\title{
INTEGRAÇÃO DE SIMULADOR ACÚSTICO EM FERRAMENTA DE MODELAGEM BIM
}

\section{INTEGRATING ACOUSTIC SIMULATOR IN BIM MODELING TOOL}

\author{
José Luis Menegotto ${ }^{1}$ \\ Escola Politécnica. Universidade Federal do Rio de Janeiro, Rio de Janeiro, RJ, Brasil, jlmenegotto@poli.ufrj.br \\ Julio Cesar Boscher Torres² \\ Escola Politécnica. Universidade Federal do Rio de Janeiro, Rio de Janeiro, RJ, Brasil, julio@poli.ufrj.br
}

\begin{abstract}
Resumo
Este artigo apresenta os resultados obtidos na primeira fase de desenvolvimento de uma aplicação que integra a interface de um ambiente de projeto BIM (Building Information Modeling) ao simulador acústico BRASS (Brazilian Room Acoustic Simulator). O objetivo do projeto é obter um ambiente de auralização integrado à ferramenta de concepção do projeto, buscando facilitar o trabalho de simulação acústica. Uma interface de auralização unificada evita que arquitetos e engenheiros tenham que comutar entre diversas aplicações gráficas para ouvir o resultado da simulação, facilitando a tomada de decisão projetual. Para efetuar a auralização de salas, os procedimentos de cálculo utilizados pelo simulador acústico BRASS foram implementados no aplicativo de modelagem BIM Revit, testando duas implementações: scripts programados no ambiente de programação visual Dynamo e funções programadas em C\# no ambiente. .NET. Como resultado da integração, são obtidos arquivos de áudio biauriculares que consideram a espacialidade do som através das funções de transferência relativas à cabeça e a visualização da posição da fonte sonora e do campo acústico, definido pelo conjunto de raios sonoros que se propagam dentro do ambiente simulado. São apontados problemas referentes ao desempenho necessário para realizar este tipo de simulações e relacionados à classificação dos parâmetros utilizados.
\end{abstract}

Palavras-chave: BRASS. Simulação acústica. Building Information Modeling. Auralização.

\begin{abstract}
This paper presents the results obtained at the first phase of the development of an application that integrates an existing auralization tool called BRASS (Brazilian Room Acoustic Simulator) into a popular BIM tool. The goal of the project is to obtain an integrated acoustical simulation tool to facilitate the design and decision-making process for the project of room acoustics. A unified and integrated interface avoids the need of architects and engineers to switch between applications to hear the simulation results, facilitating the design decisions. Procedures implemented by BRASS were reprogrammed in Revit to perform the auralization of rooms. The application was programmed on two environments: Dynamo scripts and a set of classes and macros programmed in C\# language inside the .NET environment. The results of such integration are binaural audio files that consider the sound spatiality through head-related transfer functions, as well as the visualization of the sound source location and the acoustic field defined by the sound rays, which propagate inside the simulated environment. The article also highlights issues concerning the performance required by acoustical analysis and some aspects regarding the data classification of the parameters used in the process.
\end{abstract}

Keywords: BRASS. Acoustic simulation. Building Information Modeling. Auralization.

How to cite this article:

MENEGOTTO, J. L.; TORRES, J. C. B. Integração de simulador acústico em ferramenta de modelagem BIM. PARC Pesquisa em Arquitetura e Construção, v. 10, p. e019020, 28 maio 2019 DOI:https://doi.org/10.20396/parc.v10i0.8653934 


\section{Introdução}

Medições e análises bibliométricas recentes apontam que o uso de programas de modelagem BIM (Building Information Modeling), assim como a pesquisa relacionada ao tema, vêm se consolidando dentro do campo de desenvolvimento de projeto de edificações, apresentando um crescimento significativo durante a última década (SANTOS; COSTA; GRILO, 2017; YALCINKAYA; SINGH, 2015). Nos modelos criados com esta família de programas, a existência de uma categorização bem definida dos elementos constitutivos é um fator fundamental, sem o qual as tarefas destinadas ao processamento dos dados podem ficar sujeitas a todo tipo de ineficiências. Como é destacado pela Norma Brasileira NBR 12006-2 (ABNT, 2018), "é necessária uma abordagem abrangente, completa e consistente" para a classificação de objetos da edificação. A consistência de um sistema de classificação ajudará a mapear e relacionar os dados, permitindo que, em cada etapa do processo, possam ser transformados em informações úteis e confiáveis, que auxiliem no momento da tomada de decisão dos projetistas. No estágio atual de desenvolvimento da tecnologia BIM, coexistem diversas estruturas de informação e sistemas de classificação, que objetivam sistematizar o ordenamento necessário para que o conjunto de propriedades, valores e relacionamentos dos processos, agentes, dispositivos e componentes da indústria da construção possam ser equacionados e comunicados de maneira eficiente.

\section{BuildingSMART e a Web Semântica}

O modelo de dados IFC (Industry Foundation Classes) foi originalmente projetado em esquemas escritos em linguagem EXPRESS (PAUWELS et al., 2017; PAUWEL; TERKAJ 2016; BUILDINGSMART, 2019). Desde a metade da década de 1990, o IFC vem sendo desenvolvido ininterruptamente pela organização buildingSMART, anteriormente denominada International Alliance for Interoperability (IAI). O IFC é desenvolvido a partir de consensos entre empresas produtoras de aplicações dirigidas ao mercado de Arquitetura, Engenharia e Construção (AECO), além de agentes que atuam nesse setor (BULDINGSMART, 2019). Materializado como um meio neutro, universal e aberto de dados estruturados, tem como principal objetivo funcionar como um instrumento de intercâmbio entre os modelos virtuais gerados pelas diversas aplicações disponíveis para projetos de edificações (BULDINGSMART, 2019; PAUWELS et al., 2017; CORREA; SANTOS, 2014; VENUGOPAL et al., 2012; PAZLAR; TURK, 2008). Beetz, Leeuwen e Vries (2009) destacaram que o IFC poderia ser considerado uma ontologia incompleta, pois, apesar de contar com definições conceituais dentro de um domínio de conhecimento compartilhado, carece de definições e de relacionamentos axiomáticos, elementos necessários para caracterizar ontologias passíveis de serem integradas a sistemas artificiais de raciocínio. Outros pesquisadores apontaram falhas de clareza semântica na definição dos esquemas do IFC (VENUGOPAL et al., 2012). Com o intuito de aperfeiçoar esse importante meio de intercâmbio, a organização buildingSMART trabalha continuamente em várias frentes, procurando adaptar os esquemas do IFC para novas exigências. Grupos de trabalho da organização estão focados nas definições para as futuras versões e módulos do IFC. Alguns projetos objetivam ampliar e integrar o IFC com esquemas e interfaces de infraestrutura urbana e Sistemas de Informação Geográfica (SIG). Os projetos denominados IFC-Bridges, IFC-Roadmaps e IFC-Alignment fazem parte de um esforço realizado em conjunto com a Open Geospatial Consortium (OGC), organização semelhante a buildingSMART, dedicada a formular as estruturas de dados abertos utilizadas pelos Sistemas de Informação Geográfica, dentro do domínio do planejamento urbano e territorial (BUILDINGSMART, 2019; OPEN GEOSPATIAL CONSORTIUM, 2017). Alguns esquemas com definições de objetos referentes à infraestrutura urbana começaram a ser incorporados nas versões IFC 4.1.0.0 e IFC 
4.2.0.0. A versão consultada durante o trabalho de integração do simulador acústico foi o IFC4 Adendo 2 (4.0.2.0), que trata da formalização descritiva de definições conceituais no domínio da edificação.

Destacamos aqui outra frente de ação, o projeto denominado IFC-Linked Data (dados conectados), que na última década, tem sido desenvolvido com o objetivo de definir uma camada ontológica para o IFC. O projeto de dados conectados, escrito na linguagem Ontology Web Language (OWL), adiciona os relacionamentos lógicos e axiomáticos necessários para permitir a efetiva integração do IFC com sistemas inteligentes de processamento, distribuídos em redes e dentro do paradigma de Web Semântica (BUILDINGSMART, 2019). Web Semântica é uma tecnologia conceituada por Tim Berners-Lee (2018) e regulada pela organização World Wide Web Consortium (W3C, 2019; PAUWELS et al., 2018). Pauwels et at. (2017), desenvolvedores do projeto IFCLinked Data, destacam que a interoperabilidade, a vinculação de dados entre domínios e a inferência de provas lógicas seriam três aspectos chaves da incorporação do IFC dentro do paradigma da tecnologia da Web Semântica. Em paralelo a todos os esforços de desenvolvimento de padrões abertos para intercâmbio, comunicação e processamento inteligente de dados, as empresas produtoras de aplicações continuam ampliando e adaptando os modelos de informação proprietários sobre os quais operam os seus produtos.

\section{Sistemas de classificação}

Os sistemas de classificação configuram outro instrumento de organização. Eles são promovidos e definidos por órgãos normativos ou por agências da sociedade civil - como o Construction Specifications Institute (CSI) dos Estados Unidos ou a Associação Brasileira de Normas Técnicas (ABNT). Tais sistemas têm como o objetivo organizar os dados por meio de critérios taxonômicos que permitam mapear a informação gerada pelos processos envolvidos durante o projeto da edificação, levando em consideração todo o ciclo de vida de uma construção. A ABNT, por exemplo, nos princípios metodológicos da norma referente a sistemas de classificação da construção, declara:

\footnotetext{
"Estabelecendo-se essa inter-relação entre as classes e os princípios de especialização, esta norma propõe uma estrutura de códigos, para que, de maneira ordenada, seja possível itemizar os grupos, componentes, etapas, funções, tipos e subtipos dos sistemas propostos" (ABNT, 2011, p.5).
}

Durante o processo de modelagem, os modelos BIM são alimentados com dois tipos básicos de dados: os geométricos, explicitamente declarados ou implicitamente presentes nos elementos modelados (comprimentos, áreas, alturas, contornos, etc.), e uma variedade de dados não geométricos, associados aos elementos constitutivos do projeto. Independentemente da fase ou do grau de detalhamento e desenvolvimento do projeto, para aproveitar a riqueza das informações incorporadas aos modelos, é desejável que as estruturas de informação sejam claras e completas, de forma a permitir que as operações de busca, inclusão, extração e sincronização possam ser realizadas de modo direto, sem ter que passar por sucessivos filtros ou tradutores intermediários, ganhando-se eficiência no processo. A quantidade, organização e nomenclatura dos dados dependerão do tipo de informação que se deseja gerenciar: concepção, orçamento, controle de obra, operação ou manutenção.

\section{BIM e a acústica de salas}

No que tange ao processamento acústico, pode ser verificado que o processo tradicional adotado para projetar e avaliar as qualidades acústicas de salas consiste, de um modo geral, no uso de dois tipos de programas: a literatura destaca que, em primeiro 
lugar, é construído o modelo geométrico com algum programa de modelagem e, na sequência, é transferida a geometria para os simuladores especializados como o RAVEN, ODEON, CATT-Acoustic, EASY, RenderAIR ou Ecotect (SUNYOUNG; COFFEEN; SANGUINETTI, 2013). As técnicas de transferência podem envolver operações de exportação e importação por meio de formatos de intercâmbio como o tradicional DXF ou em formatos de compartilhamento como o Green Building XML (gbXML) (SUNYOUNG; COFFEEN; SANGUINETTI, 2013; ARETZ; VORLÄNDER, 2014; SAVIOJA; SVENSSON, 2015). Savioja e Svensson (2015) mencionam que a explosão da pesquisa em simulação acústica computacional aconteceu na década de 1990, junto com o surgimento dos simuladores computacionais ODEON, CATT-Acoustic e EASY, ainda hoje utilizados pelos projetistas.

Em um levantamento comparativo entre métodos de simulação visual e métodos de simulação acústica de salas, Charalampous e Michael (2014) apontam três diferenças significativas entre o tratamento computacional dos fenômenos associados com a luz e com o som. A primeira diz respeito à natureza da luz, que sendo uma radiação eletromagnética, pode se propagar no vazio, enquanto o som, sendo considerado uma flutuação de pressão, sofre os efeitos das variações do meio no qual se propaga. A segunda diferença está relacionada aos comprimentos de onda, que na luz visível estão na faixa de 430 a $790 \mathrm{THz}$ e, no som, acontecem em frequências mais baixas, de 20 a $20000 \mathrm{~Hz}$. Finalmente, destacam a velocidade de propagação, que é de $299.794 .458 \mathrm{~m} / \mathrm{s}$ para a luz e $343 \mathrm{~m} / \mathrm{s}$ para o som. A velocidade do som, mais lenta, exige que a variável temporal seja considerada no cálculo como um fator significativo, pois os ouvintes poderão receber informações sonoras relevantes com retardo. Os três fatores tornam o cálculo da simulação sonora um processo de custo computacional elevado (CHARALAMPOUS; MICHAEL, 2014). Embora estes fatores não cheguem a explicar totalmente os motivos pelos quais a simulação acústica ainda não foi integrada aos programas de modelagem de projetos, sendo tratada com ferramentas externas especializadas, eles contribuem para compreender parte do problema.

Uma outra observação, estimulada pela leitura de análises bibliométricas sobre o tema BIM, é a quase ausência de palavras ou expressões relacionadas com o tema da acústica, como "acoustic", "sound", "impact noise", "reverberation", "vibration", "absorption" ou "resonance" nos resultados dessas análises. No levantamento de Santos, Costa e Grilo (2017), que contabilizaram 381 artigos dedicados à temática BIM entre 2005 e 2015, a palavra "acoustic" aparece apenas uma vez, vinculada a um estudo conduzido por Pauwels (PAUWELS et al., 2011). Pauwels utiliza o desempenho acústico como estudo de caso para exemplificar, dentro do contexto do projeto IFC Linked Data, uma forma de relacionar o IFC a linguagens que permitam construir regras lógicas de processamento, como o Semantic Web Rule Language (SWRL), necessárias para desenvolver semânticas aplicadas à verificação de problemas de projeto. Em outra pesquisa bibliográfica sistemática, conduzida por Yalcinkaya e Singh (2015), foram processados estudos sobre BIM escritos entre 2004 e 2014, totalizando 975 artigos ordenados em 90 níveis temáticos de classificação. Nessa pesquisa não se encontrou nenhuma palavra nem resultado sobre temas específicos de acústica. Já no Brasil, uma pesquisa bibliográfica que filtrou 409 publicações relacionadas à temática BIM entre 2002 e 2015, apontou que os temas de estudo abordados mais recorrentes dizem respeito a problemas de gestão da construção, de interoperabilidade e de processo de projeto (MACHADO; RUSCHEL; SCHEER, 2017).

$\mathrm{Na}$ atualidade, existem algumas iniciativas que integram cálculos acústicos nos programas de modelagem BIM (WU; CLAYTON, 2013), mas os simuladores especializados ainda desempenham um papel importante no domínio do cálculo 
acústico de salas. Os resultados (parâmetros acústicos, respostas impulsivas) dos diversos tipos de análises acústicas podem ser formatados em tabelas, gráficos de frequência, espectrogramas ou arquivos de áudio. O processo de análise acústica do espaço e geração de resultados ocorre em etapas, pois as ferramentas de modelagem possuem os recursos para inserir os dados e manipular geometricamente o modelo, enquanto os simuladores detêm os mecanismos e os métodos de cálculo para simular a propagação e o comportamento do som no espaço. Algumas experiencias foram realizadas exportando o modelo BIM em formato IFC para programas externos especializados no processamento de simulações, como o estudo relatado por Tan et al. (2017) relacionando o modelo de Revit com o ambiente de simulação COMSOL Multiphysics.

Uma preocupação dos pesquisadores, desenvolvedores e usuários finais tem sido estabelecer um fluxo de trabalho que minimize o processo de intercâmbio de informações entre os aplicativos de modelagem geométrica e de análise acústica (SUNYOUNG; COFFEEN; SANGUINETTI, 2013). Um fluxo de trabalho sujeito a constantes operações de tradução é apontado como uma das principais causas que dificultam a adoção deste tipo de análise, principalmente durante as etapas iniciais de desenvolvimento de um projeto (SUNYOUNG; COFFEEN; SANGUINETTI, 2013; TAN et al. 2017; WU; CLAYTON, 2013). Além disso, as aplicações de modelagem geométrica utilizadas pelos projetistas podem carecer das ferramentas que facilitem a interoperabilidade e a comunicação entre programas, obrigando-os a trabalharem em diversas aplicações e ambientes computacionais, que nem sempre possuem estruturas de dados compatíveis, requerendo trabalhos adicionais de ajuste.

Dentre os esforços que vem sendo realizados no sentido de integrar programas de modelagem de projeto à problemática de acústica de salas, pode ser citado um plug-in desenvolvido para SketchUp (ASPÖCK et al., 2014). No sistema desenvolvido, o usuário pode interagir com o ambiente movendo fontes sonoras e receptores, além de alterar os materiais das superfícies para reproduzir a resposta do som em tempo real. Outra contribuição nesse sentido é a de Wu e Clayton (2013) que programaram uma aplicação em Revit para analisar o comportamento acústico de espaços arquitetônicos por meio de dois parâmetros: o tempo de reverberação e o nível de intensidade sonora. Embora não seja dedicada à acústica de salas, podemos destacar a contribuição de (SILVA et al., 2017) que propuseram uma aplicação em Revit, desenvolvida com scripts em Dynamo, para auxiliar os projetistas a cumprirem com as condições de isolamento acústico exigidas pela norma de desempenho brasileira NBR 15575-4. Poucas iniciativas visaram integrar ambientes de modelagem de projetos aos ambientes e às técnicas aplicadas no campo da simulação acústica de salas, principalmente com renderização sonora em tempo real (ASPÖCK et al., 2014).

O presente artigo apresenta o processo de integração de uma ferramenta de modelagem BIM com a ferramenta de simulação acústica denominada BRASS. Programou-se uma aplicação (API) para incorporar a ferramenta de simulação à ferramenta de modelagem. O objetivo da API é integrar processos de trabalho, aproximando a concepção do projeto da problemática do tratamento acústico, por meio da unificação das ferramentas envolvidas, além de detectar problemas envolvidos no processo.

\section{Método}

O estudo acústico desta experiência refere-se apenas ao problema do condicionamento acústico de salas tratado pela NBR 12.179/1992, no que diz respeito à simulação do som 
propagado dentro de ambientes (ABNT, 1992). Os cálculos necessários para o tratamento do isolamento acústico não foram considerados.

Com BRASS pode-se realizar o cálculo dentro de recintos fechados ou espaços urbanos delimitados. O parâmetro fundamental utilizado neste processo é a propriedade denominada "Coeficiente de absorção acústica", para a qual foram procurados os valores disponíveis na tabela publicada pela NBR 12.179/1992 (ABNT, 1992) como também as suas denominações dentro dos sistemas de classificação normativos. Para alocar os valores dos coeficientes de absorção sonora foram analisados três domínios necessários: o ambiente de modelagem Revit v.2017; a estrutura de dados IFC4 Adendo 2 (BUILDINGSMART, 2019), necessário para realizar operações de interoperabilidade; e, finalmente, o sistema de classificação de objetos da construção da norma brasileira NBR 12.006-2 (ABNT, 2018).

\section{Módulo de simulação acústica}

O simulador BRASS foi inicialmente concebido para operar apenas como um núcleo ou engenho isolado, capaz de processar as informações acústicas e geométricas armazenadas em um arquivo texto, com blocos de informações semelhantes ao HTML e ao XML, a fim de organizar os resultados (áudios, parâmetros acústicos e respostas impulsivas) em arquivos específicos. Na primeira versão deste simulador, nenhuma interface de modelagem geométrica foi desenvolvida. Esta etapa era realizada diretamente no arquivo texto ou por meio da importação de dados dos arquivos em formato DXF. A integração com ambientes de modelagem como o Revit eliminou a etapa de conversão, permitindo acelerar o processo de verificação da resposta acústica de um projeto.

Os principais elementos da simulação utilizados em BRASS são:

- as fontes sonoras;

- os receptores;

- as superfícies que definem o tipo de geometria do ambiente simulado.

Cada um desses elementos deve conter suas respectivas propriedades acústicas. A fonte sonora é caracterizada pela sua posição espacial, pela potência e pela direção de distribuição da energia sonora (padrão de direcionalidade). O receptor (cabeça) é definido por uma posição espacial, uma orientação e um raio de influência, dentro do qual a energia dos raios é acumulada conforme os respectivos tempos de propagação. Os raios emitidos pela fonte percorrem o espaço e dissipam a energia de acordo com os coeficientes de absorção acústica das superfícies que eles atingem. A análise de cada raio é realizada de maneira independente para nove bandas de frequência de oitavas de $63 \mathrm{~Hz}$ a $16 \mathrm{kHz}$, para as quais devem ser conhecidos os coeficientes de absorção.

BRASS realiza o cálculo das respostas acústicas (respostas ao impulso) da geometria dos ambientes por meio da técnica de traçado de raios. Neste processamento, a resposta impulsiva monoaural, equivalente à resposta capturada por um microfone omnidirecional, é obtida pela soma das contribuições energéticas de cada raio no receptor. A resposta biauricular, equivalente à resposta dos ouvidos humanos, é obtida considerando, além da energia dos raios, a direção de incidência do raio na cabeça e as respectivas funções de transferência relativas à cabeça. A técnica permite gerar sinais de áudio biauriculares, os quais simulam a sensação de espacialidade do som ao redor do receptor (cabeça). É importante destacar que as três estruturas de dados participantes na experiência, isto é, a do programa Revit, o IFC e o sistema de classificação da ABNT, carecem dos campos necessários para alocar os parâmetros requeridos por BRASS para realizar o cálculo da simulação acústica no modelo virtual. 
Foi necessário recorrer ao programa Excel para poder organizar e registrar todos os valores desses parâmetros em uma planilha, além de programar as funções de sincronização entre as duas aplicações.

\section{Integração BRASS-Revit}

No processo de integração, o método de traçado de raios utilizado em BRASS foi implementado diretamente em Revit, com o objetivo de compreender visualmente os caminhos percorridos pelo som durante sua propagação entre as fontes e os receptores. A função para traçado de raios é um recurso já implementado na biblioteca de desenvolvimento de API de Revit. Ela permite efetuar o cálculo geométrico do caminho percorrido pelas sucessivas reflexões que sofre um raio lançado em espaços delimitados. $\mathrm{Na} A P I$, o traçado dos raios pode ser realizado com ou sem representação gráfica. Para poder visualizar o resultado, os raios precisam ser representados por segmentos de reta. Estes podem ser materializados e terem as suas propriedades definidas (ponto inicial, ponto final, cor e espessura). Para isso, são utilizadas diversas outras funções que complementam a biblioteca de desenvolvimento. São funções de desenho, que possibilitam a modelagem dos caminhos e permitem ter a percepção visual das perdas de energia dos raios durante o seu percurso. A integração foi programada respeitando o método de cálculo do simulador BRASS, adicionando a representação gráfica dentro do modelo 3D com a API de Revit. O processo total foi dividido em duas etapas:

1) Cálculo das perdas de energia dos raios para obter a resposta impulsiva (respeitando o método de BRASS);

2) Materialização gráfica no modelo 3D (com API Revit).

Devido às operações de visualização dos raios, a segunda etapa consome a maior parte do tempo de processamento. Nesta versão, foi implementado um mecanismo de disparo de raios omnidirecional, de modo a permitir a emissão uniforme da energia sonora para qualquer direção do espaço. Em BRASS, qualquer padrão de direcionalidade pode ser obtido mantendo os raios distribuídos uniformemente, mas alterando a energia inicial conforme a direção. Durante o processo de emissão, os raios são rastreados individualmente para cada nova reflexão. A quantidade de reflexões é um parâmetro que pode ser ajustado. Quando a distância da perpendicular entre o ponto central do receptor e a reta que define um raio lançado é menor ou igual à distância de influência definida para o receptor (tipicamente de 20 a $50 \mathrm{~cm}$ ), as informações desse raio são armazenadas em um arquivo de texto externo. Cada linha desse arquivo corresponde a um raio do qual é registrado: o tempo de propagação entre a sua emissão e o momento que atingiu o receptor; a energia em cada banda de oitava e a direção de incidência em relação ao receptor. $O$ arquivo de texto contendo essas informações de percurso corresponde ao campo acústico da posição do ouvinte. Elas são enviadas ao módulo do BRASS responsável por computar as respostas impulsivas e gerar os respectivos áudios. A análise por bandas de oitava exige que os materiais e os objetos utilizados no modelo BIM possuam um coeficiente de absorção acústica discriminado para cada banda de frequência. Na estrutura de dados dos modelos Revit existe um campo denominado Absorptance que se relaciona à problemática térmica, utilizado para alocar o índice de absorção da radiação. Como foi relatado pelas pesquisas consultadas (COSTANTINO et al., 2017; ILHAN; YAMAN, 2015; WU; CLAYTON, 2013) e observado diretamente nesta experiência, na biblioteca de materiais do programa ainda não existem campos destinados para alocar coeficientes acústicos. Já no índice oficial do esquema de informação IFC4 Adendo 2 foram encontrados os seguintes campos que permitem alocar algumas propriedades acústicas (BUILDINGSMART, 2019): 
IfcSoundPowerLevelMeasure, IfcSoundPowerMeasure, IfcSoundPressureLevelMeasure, IfcSoundPressureMeasure, PEnum_SoundScale, Pset_SoundAttenuation e Pset_SoundGeneration.

Não foram encontrados, no entanto, campos específicos para alocação dos coeficientes de absorção acústica dos materiais, referentes à perda de energia que a onda sonora sofre ao atingir um obstáculo. Tampouco foram achados no sistema de classificação Omniclass campos específicos de absorção acústica para todas as frequências necessárias, nem no sistema de classificação homólogo, publicado pela agência brasileira ABNT. Na tabela 49 do sistema Omniclass constam as seguintes propriedades: Acoustical Absorption 49.81.81.19, Sound Absorption 49.81.81.21 e Sound Absorption Average (SAA) 49.81.81.23 (CONSTRUCTION SPECIFICATIONS INSTITUTE, 2019). No sistema da ABNT, definido na norma NBR 15.965-2 (ABNT, 2012), essas três propriedades encontram-se classificadas nos campos Absorção acústica OP.60.90.05, Absorção sonora OP.60.90.07 e Média de absorção sonora (SAA) OP.60.90.07. Como o processo de simulação acústica visa à obtenção de respostas cada vez mais precisas da propagação do som e sua percepção dentro dos ambientes, foi necessário alocar todos os coeficientes de absorção envolvidos no processamento de BRASS em arquivos externos ao modelo, utilizando um campo alfanumérico como chave de acesso a uma tabela Excel, conforme ilustrado na Tabela 1. Para o preenchimento desta tabela, foram utilizados os coeficientes da norma ABNT NBR 12.179:1992 (ABNT, 1992). Como a norma fornece coeficientes para seis frequências, nas faixas compreendidas entre $125 \mathrm{~Hz}$ a 4 $\mathrm{kHz}$, foi decidido complementar as faixas faltantes repetindo os coeficientes extremos ( $63 \mathrm{~Hz}, 8 \mathrm{kHz}$ e $16 \mathrm{kHz}$ ). Cada registro da tabela Excel tem um campo com o código do material e seus coeficientes de absorção correspondentes. Para realizar a associação entre os coeficientes de absorção e os objetos do modelo foi utilizado o campo Type Mark, já que, por ser um campo de identidade relacionado ao tipo do componente, está presente em todos os objetos construtivos, além de permitir a alocação de dados tipo string. A seguir é apresentado o código para extração dos coeficientes:

$$
\begin{aligned}
& \text { D_excel = Excel.ReadFromFile(arquivo, "Coeficientes", false); } \\
& \text { tipo = elemento.GetParameterValueByName("Type"); } \\
& \text { codi = FamilyType.GetParameterValueByName(tipo, "Type Mark"); } \\
& \text { codi2 = List.AddltemToFront("Aoo", codi<1>); }
\end{aligned}
$$

Estuda-se, ainda, a possibilidade de relacionar a tabela de coeficientes de absorção ao campo Keynote para permitir integrar os coeficientes acústicos ao sistema de classificação de orçamento. Esta opção, que visa à unificação das fontes de informação, está em fase de análise.

Tabela 1 - Coeficientes de absorção acústica da NBR 12.179 (ABNT, 1992) com colunas extremas repetidas (63 Hz, 8 kHz e 16 kHz).

\begin{tabular}{llccccccccc}
\hline & & $\mathbf{H z}$ & $\mathbf{H z}$ & $\mathbf{H z}$ & $\mathbf{H z}$ & $\mathbf{k H z}$ & $\mathbf{k H z}$ & $\mathbf{k H z}$ & $\mathbf{k H z}$ & $\mathbf{k H z}$ \\
\hline Cod. & Material & $\mathbf{6 3}$ & $\mathbf{1 2 5}$ & $\mathbf{2 5 0}$ & $\mathbf{5 0 0}$ & $\mathbf{1}$ & $\mathbf{2}$ & $\mathbf{4}$ & $\mathbf{8}$ & $\mathbf{1 6}$ \\
\hline $\mathbf{A 0 1}$ & Taco colado & 0,04 & 0,04 & 0,04 & 0,06 & 0,12 & 0,10 & 0,17 & 0,17 & 0,17 \\
\hline $\mathbf{A 0 2}$ & Reboco áspero, cal & 0,03 & 0,03 & 0,03 & 0,03 & 0,03 & 0,04 & 0,07 & 0,07 & 0,07 \\
\hline A03 & Vidro plano $3 \mathrm{~mm}$ & 0,23 & 0,23 & 0,11 & 0,09 & 0,01 & 0,01 & 0,03 & 0,03 & 0,03 \\
\hline $\mathbf{A 0 4}$ & Forro de gesso & 0,04 & 0,04 & 0,04 & 0,08 & 0,12 & 0,03 & 0,10 & 0,10 & 0,10 \\
\hline
\end{tabular}

Fonte: Adaptada da NBR 12.179 (ABNT, 1992).

\section{Resultados}

A aplicação foi implementada para funcionar dentro do ambiente de modelagem do programa Revit. Foram escritas e testadas duas implementações. A primeira foi projetada em ambiente de programação visual Dynamo, integrando algumas funções escritas em linguagem C\# no ambiente .NET. A segunda foi escrita totalmente em C\#. A 
seguir são relatadas as principais características de ambas as implementações e as causas que motivaram a reprogramação do sistema.

\section{Implementação em Dynamo}

Nesta implementação, foram programados um emissor sonoro, um receptor, um controle para configurar a quantidade de raios lançados e um controle para realizar o ajuste angular e o tamanho de painéis acústicos. Os painéis acústicos foram criados como famílias de componentes genéricos e preparados com parâmetros de dimensionamento e de inclinação. Deve-se mencionar que, apesar de serem peças importantes para a configuração arquitetônica dos espaços, eles não são essenciais para o processo de cálculo da simulação. Ela pode ser realizada com ou sem a intervenção dos painéis acústicos. Para minimizar a quantidade de interfaces e ligações no fluxograma da aplicação, optou-se por concentrar os pacotes de funcionalidades em code-blocks e grupos específicos de funções, conforme ilustrado na Figura 1.

Figura 1 - Fluxograma do processo em Dynamo

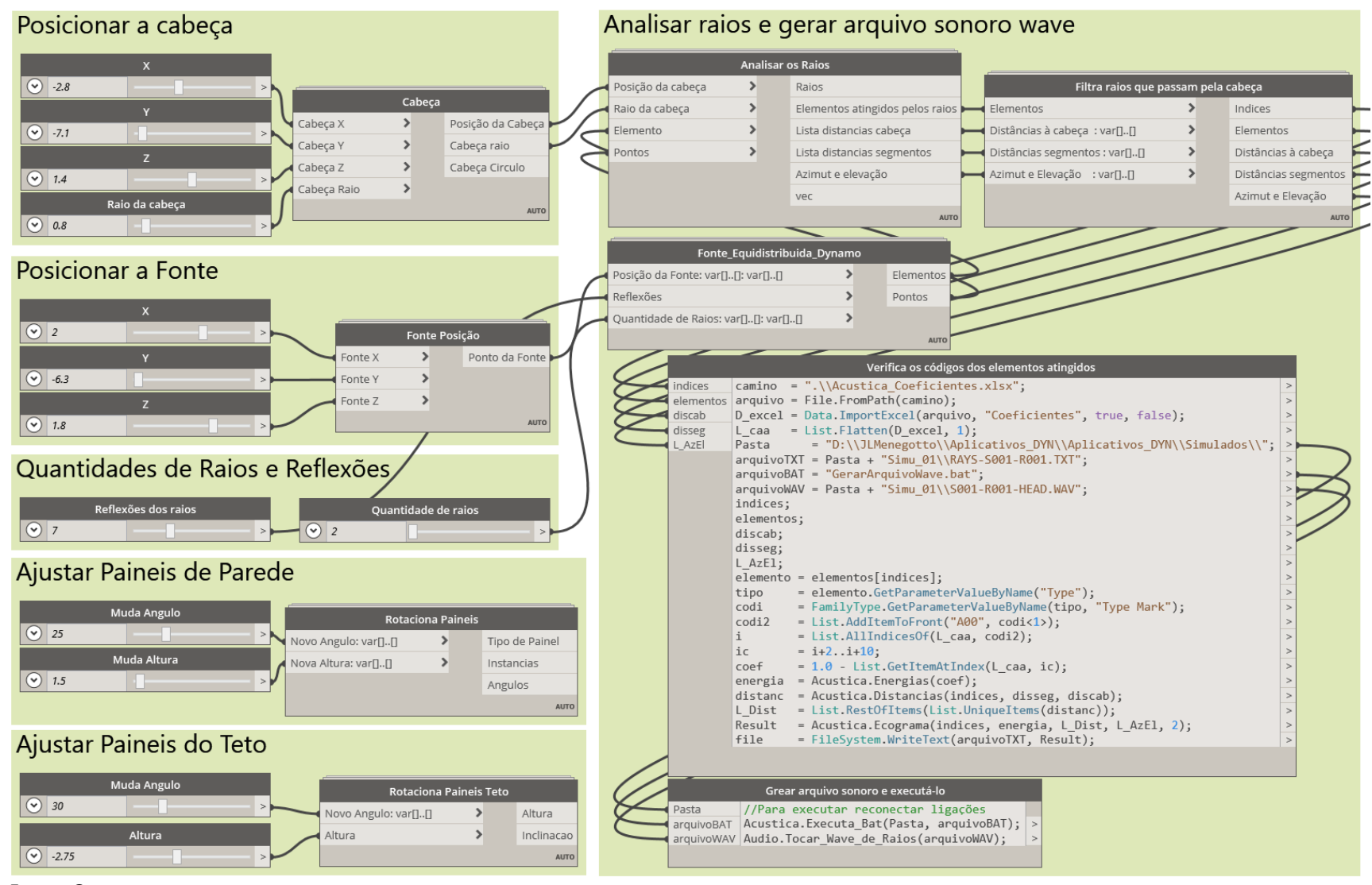

O maior problema encontrado nesta versão diz respeito ao desempenho de cálculo. Para obter resultados acústicos satisfatórios, a quantidade de raios traçados deve ser da ordem de $10^{5}$ a $10^{6}$, em função da absorção média da sala e do tempo de reverberação. Para o caso avaliado precisariam ser lançados não menos de 50.000 raios. Em Dynamo, a função que realiza o cálculo geométrico da reflexão do raio está prédefinida por uma interface que não permite discriminar quais raios serão desenhados no modelo. Por causa deste condicionante, foi necessário desenhar todos os raios, conforme mostrado na Figura 2. 


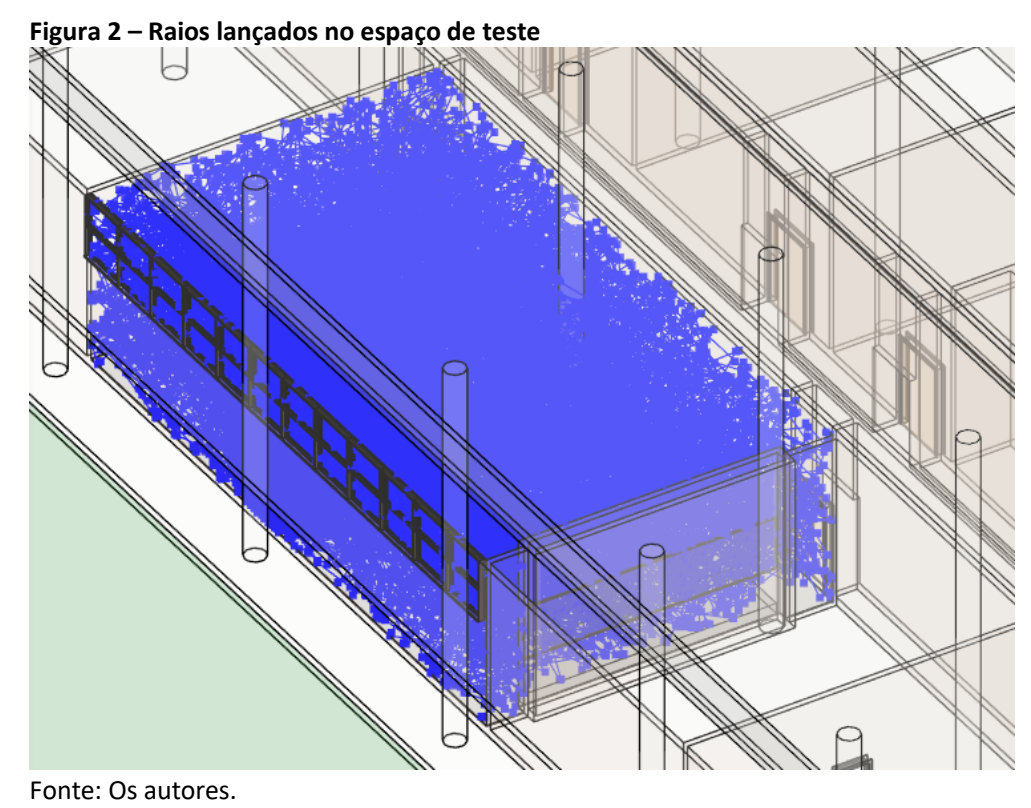

A densidade dos raios apresentados no resultado impede tanto de visualizar a sua distribuição espacial como de ter a percepção temporal das perdas de energia entre a fonte e o receptor. Observou-se também que o processo de cálculo sofre um retardo considerável devido à etapa de desenho, pois todos os raios são temporariamente apresentados no modelo, independentemente de serem ou não computados no cálculo da resposta acústica no receptor. Nos testes realizados com a interface Dynamo, o tempo de processamento obtido ao lançar 700 raios com 5 reflexões foi de 20 minutos. Portanto, considerou-se que o objetivo de calcular 50.000 raios tornaria o procedimento muito demorado, inviabilizando-o de um ponto de vista prático, por causa da demorada resposta da operação de renderização visual/sonora. Esse aspecto, relacionado à velocidade de resposta esperada e desejada para uma ferramenta de projeto, foi crucial para motivar a reprogramação do sistema, deixando de lado a interface de Dynamo. Com relação à quantidade de reflexões necessárias para construir as respostas impulsivas, o algoritmo utilizado por BRASS utiliza a sobreposição do resultado do traçado de raios com uma cauda reverberante artificial, gerada através das informações do decaimento da energia e dos coeficientes médios de absorção. Dessa forma, são necessárias poucas reflexões, cuja função é simular com melhor precisão as primeiras reflexões na sala.

\section{Implementação total em ambiente .NET}

Para aprimorar o desempenho do sistema, o processo foi reprogramado completamente em ambiente.NET, utilizando a linguagem C\#. Na nova implementação, o tempo de cálculo obtido para 50.000 raios com 5 reflexões foi de aproximadamente quatro minutos, apresentando uma melhora substancial na operação de amostragem visual dos raios da renderização sonora. Como a interface em C\# permitiu obter maior controle sobre o processo de cálculo e traçado geométrico de raios, buscou-se tornar ainda mais eficiente todo o processo, dividindo-o em duas etapas. Na primeira, foram calculados os percursos espaciais de todos os raios e computadas todas as absorções, ângulos e dados de direcionalidade necessários ao cálculo das respostas impulsivas de acordo com BRASS. Para esta tarefa, os objetos Referencelntersector e ReferenceWithContext, da biblioteca de funções API de Revit, permitiram maior controle na operação de traçado de raios. O resultado do cálculo foi armazenado em um arquivo texto ASCII, que contém, portanto, um registro completo do campo acústico, que é formado pelo conjunto de todos os raios (frentes de onda) que se propagaram dentro 
do ambiente simulado, independentemente de passarem ou não perto do receptor. $O$ campo acústico, assim definido, será constante se os parâmetros de posicionamento da fonte sonora, as suas propriedades, a disposição geométrica dos espaços e os materiais utilizados permanecerem inalterados. Esse método permite que a verificação espacial do receptor em qualquer local do campo acústico seja calculada, posteriormente, sem a necessidade de recalcular todo o traçado de raios, pois o receptor, ao se movimentar, atravessa um campo constante de feixes, sendo necessário verificar apenas quais são os raios que interceptam o volume próximo à posição do receptor. Tal verificação é feita pela leitura de todos os raios registrados do arquivo de texto, computando as distâncias relativas para a atual posição do receptor. No campo acústico de raios, cada segmento de reta ou feixe corresponde a uma das reflexões no percurso de um raio, que possui um tempo de propagação e energia sonora. Assim, é possível calcular a contribuição de energia de cada raio para o receptor. Por meio deste método, o cálculo do campo acústico do recinto é realizado somente uma vez (50.000 raios), enquanto o cálculo da resposta impulsiva é realizado quando acontece uma alteração da posição do receptor (verifica-se somente os raios que passam perto do receptor). Graças à economia de operações de processamento, esta técnica permite calcular e desenhar sequencialmente de modo mais eficiente os raios que atingem o receptor nas diversas posições que ele tem quando se desloca pelo espaço. Na Figura 3, é apresentada uma sequência na qual foi reposicionada a cabeça no campo de feixes, sendo desenhados apenas os raios que passaram perto do receptor em cada posição. Pode-se ajustar a distância de proximidade da cabeça, definindo assim uma região de influência na qual os raios são considerados. Durante o processo de cálculo, cada imagem da sequência é exportada a um arquivo de imagem no formato PNG.

Figura 3 - Sequência de traçado de raios com o receptor movimentando-se pela sala. Cada imagem mostra os feixes de raios que passam próximos ao receptor e, portanto, entram no cálculo da resposta impulsiva
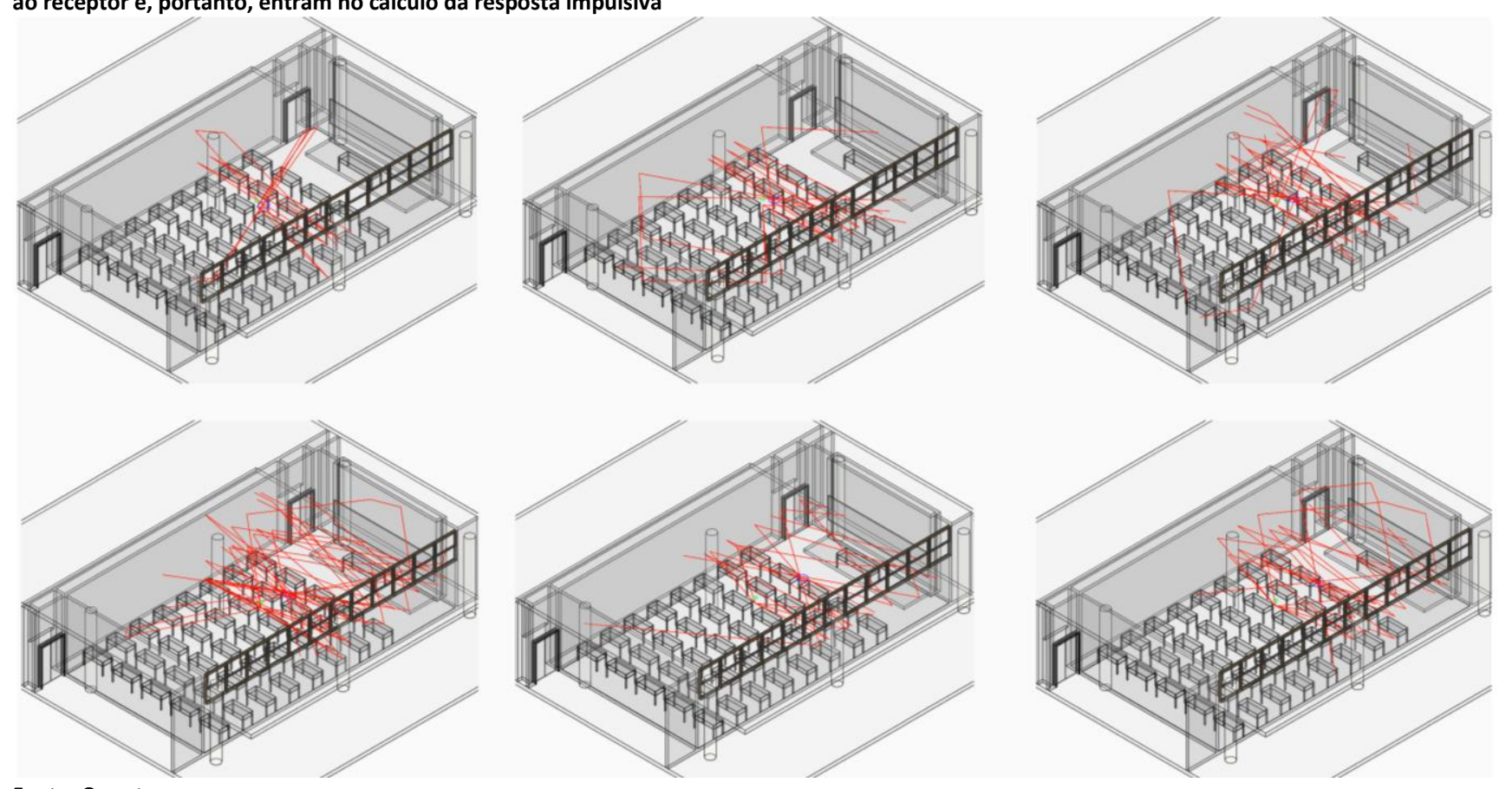

Fonte: Os autores. 


\section{Discussão}

\section{Verificação dos resultados simulados}

A incorporação do simulador BRASS ao programa Revit é apenas um dos aspectos do trabalho. Outro aspecto trata da confiabilidade dos resultados acústicos obtidos pelo cálculo realizado pelo algoritmo de BRASS, que está em constante processo de aprimoramento. Com esse objetivo, a precisão do simulador BRASS foi testada por meio de medições da resposta acústica de uma sala real, que foram comparadas às respostas extraídas pelo processo virtual, simulado, neste caso, no modelo BIM. As medições acústicas reais foram feitas no local gravando fontes sonoras distribuídas em pontos da sala previamente determinados. O ambiente utilizado é uma sala de aula com as seguintes dimensões: $L=8,20 \mathrm{~m}, \mathrm{P}=15,90 \mathrm{~m} \mathrm{e} \mathrm{H}=4,50 \mathrm{~m}$ (Figura 4). Quando a sala está ocupada somente com o mobiliário possui tempo de reverberação inadequado para a sua função $(\sim 2,2 \mathrm{~s})$. Para realizar as medições foram utilizados microfones omnidirecionais ECM 8000 para a captação e, como fonte sonora, uma caixa acústica modelo Eurolive B212 de 2 vias. Foram medidas três posições de recepção, emitindo o som desde a posição conforme ilustrado na Figura 4.

Figura 4 - Localização da fonte e dos receptores na sala utilizada como exemplo

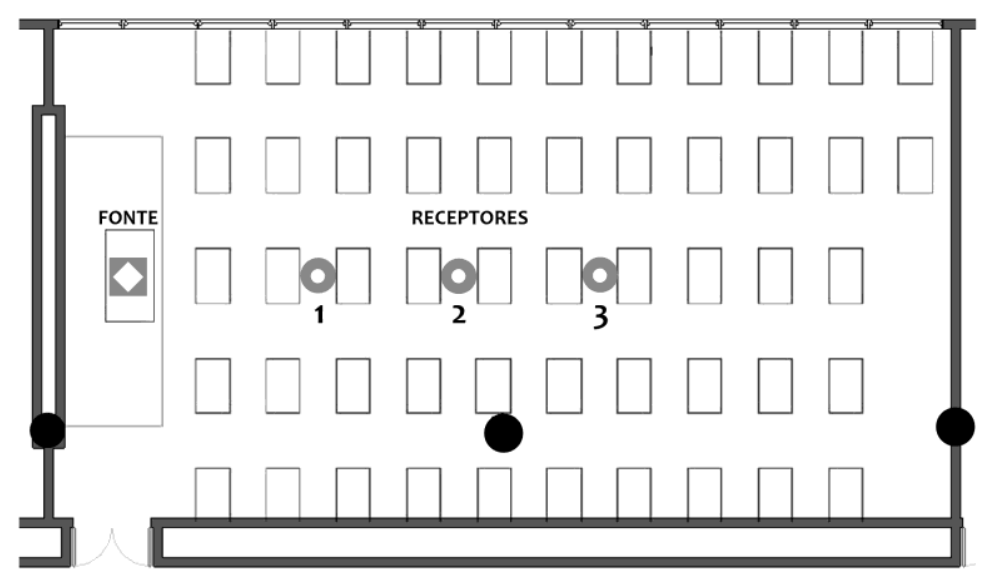

Fonte: Os autores.

Na Figura 5 são apresentadas as respostas impulsivas para o receptor 2, na qual podem ser comparados os perfis de decaimento completo de energia (Figura 5 à esquerda) e as primeiras reflexões (Figura 5 à direita) entre as respostas medida e simulada. Podem ser observadas pequenas variações nos picos de amplitude e na energia da cauda reverberante (Figura 5 à esquerda), devido principalmente a dois fatores. O primeiro é a diferença de direcionalidade entre a fonte utilizada para medição e a usada na simulação (omnidirecional). O software BRASS, na sua versão não integrada ao Revit, pode utilizar diferentes padrões de direcionalidade para as fontes, por meio de arquivos OpenDaff.

Padrões de direcionalidade ainda estão sendo implementados na versão do simulador em Revit, fazendo com que a fonte simulada tenha, ainda, somente uma característica omnidirecional. Outra causa da disparidade dos resultados diz respeito aos coeficientes de absorção acústica obtidos nas tabelas da literatura consultada. Eles podem não corresponder, exatamente, aos encontrados nos materiais do ambiente. Este é um problema recorrente em simulações acústicas, principalmente quando o tempo de reverberação é elevado e os coeficientes de absorção pequenos (da ordem de $10^{-1}$ ). 
Figura 5 - Comparação entre as respostas impulsivas medida e simulada: (à esquerda) duração total da resposta; (à direita) primeiras reflexões
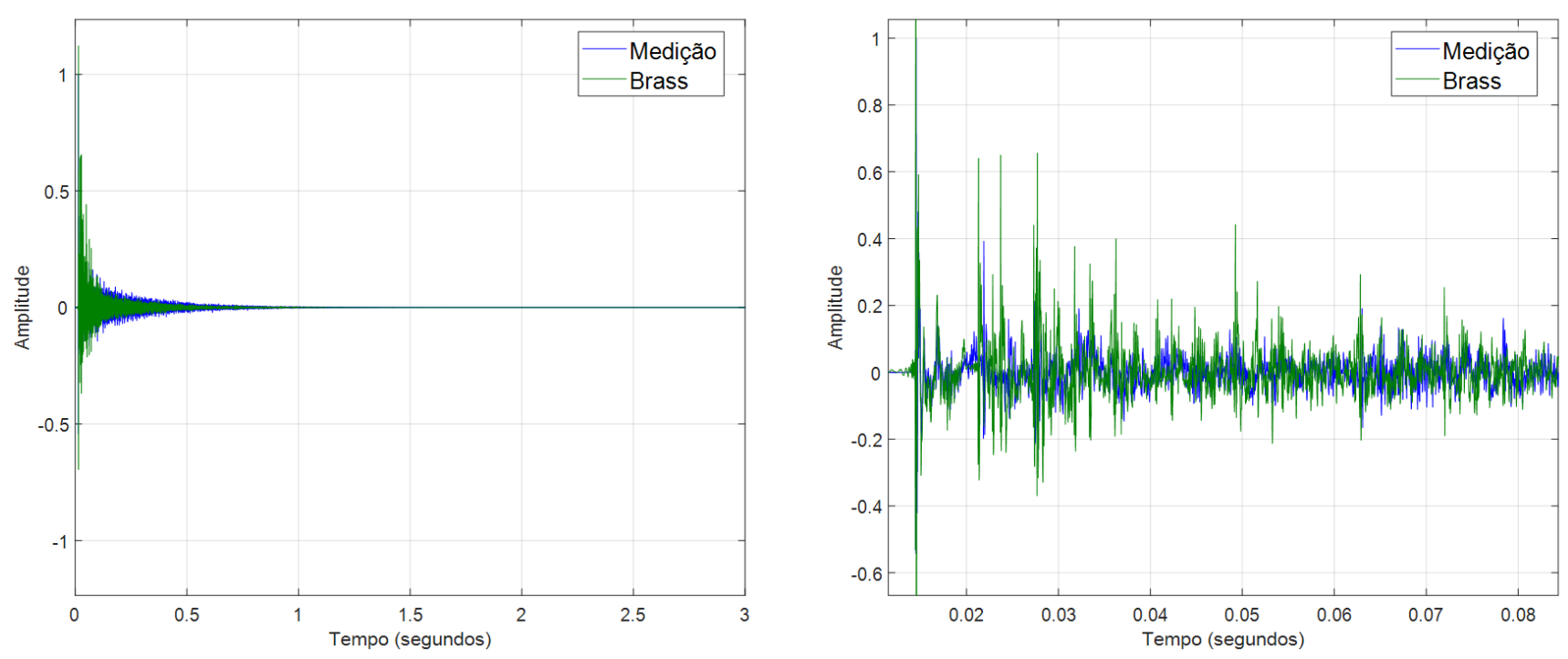

Fonte: Os autores.

Nesta versão, o mecanismo de disparo dos raios foi implementado de modo omnidirecional, simulando uma propagação sonora homogeneamente distribuída dentro do ambiente. Os gráficos mostram que não houve perdas significativas na qualidade das respostas impulsivas.

\section{Próximos passos}

Em paralelo, estudam-se mecanismos de disparo de raios direcionados, como os ilustrados na Figura 6. A imagem ilustra uma fonte cônica que dispara raios divergentes na direção vertical refletindo alternadamente no forro e no piso. O mecanismo permite controlar a abertura do foco de propagação, a densidade de raios traçados e a quantidade de reflexões.

Figura 6 - Mecanismo de disparo cônico com ajuste de foco

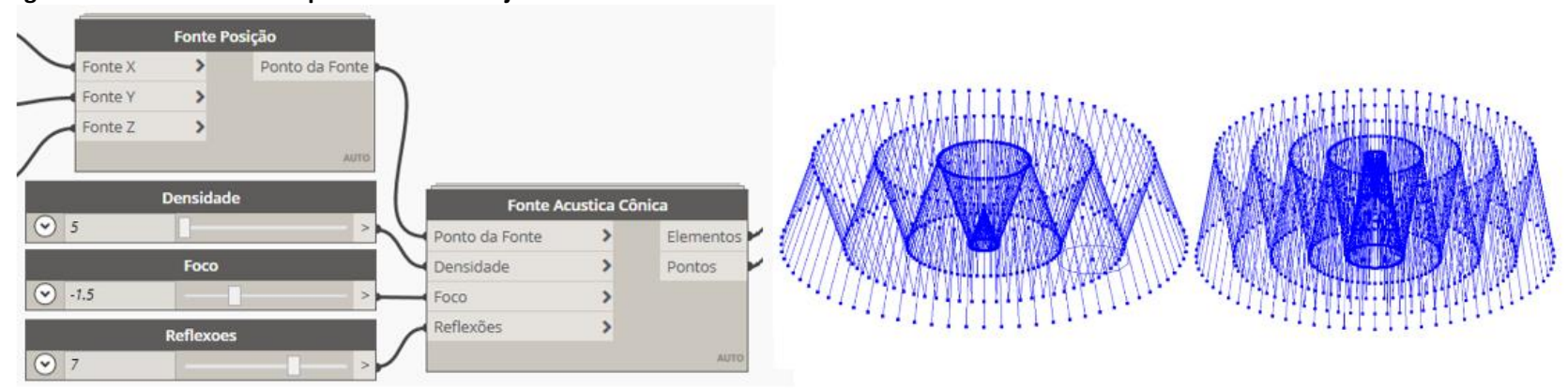

Fonte: Os autores.

Apesar de ter-se mudado o ambiente de programação de Dynamo para .NET, devido às exigências de desempenho computacional mencionadas, pretende-se continuar utilizando o ambiente de programação visual do Dynamo para realizar os primeiros testes da aplicação, graças à facilidade e rapidez que esse ambiente oferece para manipular códigos e interfaces de controle. Posteriormente, os algoritmos devem ser reescritos em ambiente. NET, no qual se consegue melhorar o desempenho de processamento.

\section{Conclusão}

Neste trabalho foi desenvolvido um módulo de integração entre uma ferramenta de modelagem de informação da edificação (BIM/Revit) com o simulador acústico BRASS. 
A fusão das ferramentas possibilitou a visualização dos raios acústicos, a geração das respostas impulsivas e a geração dos resultados auditivos (convoluções de sinais anecóicos) em arquivos de som WAV de forma interativa e integrada à ferramenta de projeto.

Um dos problemas críticos enfrentados durante a implementação da solução em forma de API, diz respeito ao uso e aproveitamento das estruturas de dados disponíveis para alocar as propriedades necessárias ao cálculo. Pazlar e Turk (2008) realizaram um estudo analítico de interoperabilidade, publicado no final da década de 2000. 0 estudo conduzido por eles apontou que, apesar das mais de 600 classes de objetos que as especificações do IFC $2 \times 3$ tinha na época, do ponto de vista do usuário final, o IFC era ainda um modelo para intercâmbio de dados insuficiente (PAZLAR; TURK, 2008). Na atualidade, BONDUEL et al. (2018) destaca que o Adendo 2 do IFC 4 tem aproximadamente 1200 classes (BONDUEL et al., 2018). Em nosso trabalho verificamos que, no que tange às propriedades acústicas envolvidas, não somente o IFC, mas as quatro estruturas de dados necessárias, utilizadas ou consultadas para realizar a integração do BRASS, isto é: a estrutura de dados proprietária sobre a qual são construídos os objetos de Revit; os esquemas de dados do IFC4 Adendo 2; o sistema de classificação da ABNT; e, finalmente, o sistema de classificação Omniclass, carecem dos campos específicos para alocar todos os dados requeridos pelo cálculo acústico implementado pelos simuladores. Como foi mencionado, o tratamento do som no simulador BRASS analisa as ondas sonoras dividindo-as em nove faixas de frequência. Tal segmentação exige que os materiais e os objetos utilizados no modelo virtual possuam um coeficiente de absorção acústica individual para cada uma dessas faixas de frequência. Constatou-se que campos descritivos para alocar esses valores são inexistentes ou insuficientes. Além disso, para simuladores que calculam também a transmissão acústica entre partições, são necessários outros conjuntos de parâmetros, por faixas de frequência, além dos coeficientes de absorção.

Outro aspecto que pode ser destacado diz respeito ao modo de publicação dos dados, especialmente no que se refere aos sistemas de classificação Omniclass e ABNT. Comparando ambos os sistemas, observou-se uma diferença importante. As tabelas Omniclass são publicadas na internet pelo Construction Specifications Institute em formatos PDF e xIsx (OMNICLASS, 2019). De modo geral, o formato xIsx permite que a incorporação de dados dentro de sistemas de processamento seja mais rápida e direta, sem que seja necessário realizar procedimentos especializados, agilizando o processo de mapeamento das informações. Já as tabelas da ABNT, publicadas apenas em formato PDF, requerem um trabalho adicional para serem integradas aos processos de mineração e automatização de dados. Nesse sentido, seguindo o conceito de pontuação ou qualificação dos dados conectados (linked data) de Tim Berners-Lee (2018), que pontua os dados publicados de acordo com um critério de cinco níveis ou estrelas, as tabelas fornecidas pela ABNT qualificariam como dados de uma estrela, enquanto as tabelas Omniclass, já estruturadas e disponibilizadas em formato proprietário xlsx, estão no patamar de dados qualificados por duas estrelas, podendo facilmente serem transformadas a um formato neutro, como o csv ou txt, colocando-as no patamar dos dados qualificados como três estrelas.

Acreditamos que esta observação seja pertinente, uma vez que no processo evolutivo dos métodos de projeto e gerenciamento digital da informação, faz-se necessário relacionar o modelo virtual com estruturas de dados cada vez mais extensas, dispersas e heterogêneas (PAUWELS et al., 2018), sem prejudicar, na medida do possível, os dados já carregados em modelos BIM existentes (BONDUEL et al., 2018). 
Os esforços atuais das entidades que organizam e promovem estruturas de dados abertos, como o BuildingSMART e o Open Geospatial Consortium, apontam para a necessidade de realizar a passagem dos processos contidos em ambientes de modelagem isolados para processos que se desenvolvam em ambientes dispersos, integrados e modularizados (PAUWELS et al., 2017). Um exemplo dessa tendência é o trabalho conjunto que promovem as duas organizações, buscando integrar os esquemas de dados abertos que existem nos domínios de conhecimento interdependentes, como o da edificação e o urbano. Os ambientes digitais de processamento, armazenamento e transporte de informação parecem haver consolidado a sua incorporação em redes e nuvens que operam intensamente no campo da Web Semântica e que, não raramente, funcionam dirigidos por sistemas autonomizados com técnicas de aprendizado de máquina (YALCINKAYA; SINGH, 2015).

O crescente volume de dados manipulados que devem ser inter-relacionados nos fluxos de trabalho envolvidos em processos de projeto em BIM, somado à heterogeneidade de formatos que participam nestes processos (PAUWELS et al., 2018), são fatores que pressionam para que seja ultrapassada a barreira dos métodos manuais de modelagem BIM. Por método manual de modelagem, entendemos aqui, o uso das aplicações tal como são fornecidas pelas empresas de software, sem nenhum tipo de adição especialmente programada para autonomizar os procedimentos de modelagem dos usuários. Nesse sentido, destacamos que o atual nome da organização buildingSMART, antiga International Alliance for Interoperability, nos sinaliza em direção de essa autonomização. Se, na sua origem, o nome da organização refletia a problemática relativa à formalização de estruturas de informação que permitissem a interoperabilidade entre programas de modelagem (BUILDINGSMART, 2019; PAZLAR; TURK, 2008), desde o ano 2008, o nome da organização passou a refletir a ampliação dessa problemática (BUILDINGSMART, 2019). O projeto de dados conectados, IFCLinked Data, posiciona claramente a tecnologia BIM no campo da Inteligência Artificial e do processamento autônomo de dados, no qual a ação humana torna-se menos necessária para os fluxos de processamento que, uma vez representados por descrições formalizadas em linguagem de máquina, podem ser tratados pelos algoritmos de raciocínio mecanizado. Todavia, para que a interpretação e transporte de dados entre sistemas de processamento seja eficiente e possam ser integrados a sistemas inteligentes robustos, beneficiando os fluxos de trabalho em BIM, é fundamental a formalização previa de ontologias abrangentes e estruturadas, de modo a relacionar os elementos participantes por Identificadores Uniformes de Recursos (Uniform Resource Identifier (URI) e descrições conceituais estruturadas (Resources Description Framework) RDF (HOR et al., 2018; PAUWELS et al., 2018; RASMUNSSEN; HVIID; KARLSHOJ, 2017;). Em outras palavras, dados que possam ser qualificados como quatro e cinco estrelas dentro do conceito de pontuação de dados conectados.

Em concordância com os esforços que vêm sendo realizados durante a última década, na direção da implementação da camada ontológica do IFC, a ifcOWL (BEETZ; LEEUWEN; VRIES, 2009; PAUWELS; TERKAJ, 2016; PAUWELS et al., 2018; BONDUEL et al., 2018), destacamos a importância estratégica que significa poder contar também com sistemas de classificação abertos e integrados à Web Semântica, dentro do paradigma de dados conectados de Berners-Lee (2018). Assim como o IFC, tais sistemas, como o da ABNT, são peças fundamentais nas quais se apoia a corrente logística dos fluxos de processos e dados que trafegam durante o ciclo de vida de uma edificação. Durante o trabalho de incorporação do simulador acústico BRASS a Revit, ficou evidente que a programação de aplicações deste tipo é altamente dependente da facilidade de acesso aos diversos, heterogêneos e dispersos fragmentos de dados necessários. 
Com relação à implementação do mecanismo traçador de raios, até o presente momento ele foi programado para que os raios sejam lançados de modo omnidirecional e com energia inicial constante. A pesquisa pretende continuar evoluindo sobre os seguintes aspectos: a direcionalidade das fontes sonoras, a dinamização do deslocamento dos receptores e a otimização do desempenho de computo, visando à obtenção de respostas simuladas em tempo real com receptores movimentando-se dentro do campo de feixes acústicos.

\section{Referências}

ABNT - ASSOCIAÇÃO BRASILEIRA DE NORMAS TÉCNICAS. NBR 12.179:1992: Tratamento acústico em recintos fechados. Rio de Janeiro, 1992.

ABNT - ASSOCIAÇÃO BRASILEIRA DE NORMAS TÉCNICAS. NBR 12.006-2: Construção da edificação - Organização de informação da construção. Parte 2: Estrutura para classificação. Rio de Janeiro, 2018.

ABNT - ASSOCIAÇÃO BRASILEIRA DE NORMAS TÉCNICAS. NBR 15.965-1:2011: Sistema de classificação da informação da construção. Parte 1: Terminologia e estrutura. Rio de Janeiro, 2011.

ABNT - ASSOCIAÇÃO BRASILEIRA DE NORMAS TÉCNICAS. NBR 15.965-2:2012: Sistema de classificação da informação da construção. Parte 2: Características dos objetos da construção. Rio de Janeiro, 2012.

ARETZ, M.; VORLÄNDER, M. Combined wave and ray-based room acoustic simulations of audio systems in car passenger compartments, Part II: Comparison of simulations and measurements. Applied Acoustics, v. 76, p. 52-65, 2014. DOI: https://doi.org/10.1016/j.apacoust.2013.07.020.

ASPÖCK, L.; PELZER, S.; WEFERS, F.; VORLÄNDER, M. A Real-Time Auralization plugin for architectural design and education. In: INTERNATIONAL AURALIZATION SYMPOSIUM, 2.; INTERNATIONAL SYMPOSIUM ON AMBISONICS AND SPHERICAL ACOUSTICS, 5., 2014, Berlin. Proceedings of the EAA Joint Symposium on Auralization and Ambisonics. Berlin: Technisch Univesitati Belin, 2014. DOI: http://dx.doi.org/10.14279/depositonce-26.

BEETZ, J.; LEEUWEN, J. V.; VRIES, B. IfCOWL: A case of transforming EXPRESS schemas into ontologies. Artificial Intelligence for Engineering Design, Analysis and Manufacturing. v. 23, n. 1, p. 89-101, 2009. DOI: https://doi.org/10.1017/S0890060409000122

BERNERS-LEE, T. Linked Data. Disponível em: https://www.w3.org/Designlssues/LinkedData.html. Acesso em: 30 nov. 2018.

BONDUEL, M.; ORASKARI, J.; PAUWELS, P.; VERGAUWEN, M; KLEIN, R. The IFC to Linked Building Data ConverterCurrent Status. In: LINKED DATA IN ARCHITECTURE AND CONSTRUCTION WORKSHOP, 6., 2018, London.

Proceedings [... ]. London: CEUR-WS, 2018. v. 2159. p. 34-43. Disponível em: http://ceur-ws.org/Vol-2159/. Acesso em: 25 mai. 2019.

BUILDINGSMART. Industry Foundation Classes Release 4 (IFC4). Disponível em: http://www.buildingsmarttech.org/ifc/IFC4/final/html/index.htm. Acesso em: 30 jan. 2019.

CHARALAMPOUS, A; MICHAEL, D. Sound propagation in 3D spaces using computer graphics techniques. In: INTERNATIONAL CONFERENCE ON VIRTUAL SYSTEM MULTIMEDIA, 20., 2014, Hong Kong. Proceedings [...]. Hong Kong: VSMM, 2014. p. 43-49.

CONSTRUCTION SPECIFICATIONS INSTITUTE. Standards. Disponível em: https://www.csiresources.org/practice/standards. Acesso em: 30 jan. 2019.

CORRÊA, F.; SANTOS, E. Ontologias na construção civil: uma alternativa para o problema de interoperabilidade com o uso do IFC. Gestão \& Tecnologia de Projetos, v. 9, n. 2, p. 7-22, 3 dez. 2014. DOI:https://doi.org/10.11606/gtp.vgi2.69141. 
COSTANTINO, C. M; BACCOLI, R; FRATTOLILLO, A; MARINI, M; DI BELLA, A; DA POS, V. The Building Information Model and the IFC Standard: Analysis of the Characteristics Necessary for the Acoustic and Energy Simulation of Buildings. In: BUILDING SIMULATION APPLICATION. 3., 2017, Bozen-Bolzano. Proceedings [... ]. Italy: BSA, 2017. p. 479-486.

HOR, A.-H; E; GUNHO, S; CLAUDIO, P; JADIDI, M; AFNAN, A. A Semantic graph database for BIM-GIS integrated information model for an intelligent urban mobility Web application. In: MID-TERM SYMPOSIUM OF THE INTERNATIONAL SOCIETY OF PHOTOGRAMMETRY, REMOTE SENSING AND SPACTIAL INFORMATION SCIENCE, 4., 2018, Deft. Annals [... ]. Deft: ISPRS, 2018, Vol. IV-4. p. 89-96. DOI: https://doi.org/10.5194/isprs-annals-IV-4-89-2018

ILHAN, B; YAMAN, H. IFC-Based Sustainable Construction: BIM and Green Building Integration. In: CIB W78 INTERNATIONAL CONFERENCE, 32., 2015, Eindhoven. Proceedings [... ]. Eindhoven: CIB W78, 2015. Disponível em: http://itc.scix.net/data/works/att/w78-2015-paper-032.pdf. Acesso em: 23 mai. 2019.

MACHADO, Fernanda Almeida; RUSCHEL, Regina Coeli; SCHEER, Sergio. Análise da produção científica brasileira sobre a Modelagem da Informação da Construção. Ambient. constr., Porto Alegre , v. 17, n. 4, p. 359-384, dez. 2017 . DOI: http://dx.doi.org/10.1590/s1678-86212017000400202.

OMNICLASS. A Strategy for Classifying the Built Environment. Disponível em: http://www.omniclass.org. Acesso em: 30 jan. 2019.

OPEN GEOSPATIAL CONSORTIUM. Future City Pilot-1: Using IFC/CityGML in Urban Planning Engineering Report. Disponível em: http://docs.opengeospatial.org/per/16-097.html\#_conclusions_and_future_work. Acesso em: 1 abr. 2019.

PAUWELS, P.; KRIJNEN, T; TERKAJ, W.; BEETZ, J. Enhancing the ifcOWL ontology with an alternative representation for geometric data. Automation in Construction. v. 80, p. 77-94, 2017.

DOI:https://doi.org/10.1016/j.autcon.2017.03.001

PAUWELS, P.; MCGLINN, K; TÖRMÄ, S.; BEETZ, J. Linked Data. Borrmann A., König M., Koch C., Beetz J. (eds) Building Information Modeling. Springer, Cham, 2018. DOI: https://doi.org/10.1007/978-3-319-92862-3_10

PAUWELS, P; TERKAJ, W. EXPRESS to OWL for construction industry: towards a recommendable and usable ifcOWL ontology. Automation in Construction. v. 63, p.100-133, 2016. DOI: https://doi.org/10.1016/j.autcon.2015.12.003

PAUWELS, P.; VAN DEURSEN, D; VERSTRAETEN, R; DE ROO, J; DE MEYER, R. A semantic rule checking environment for building performance checking. Automation in Construction. v. 20, p. 506-518, 2011. DOI:

https://doi.org/10.1016/j.autcon.2010.11.017

PAZLAR T, TURK Z. Interoperability in practice: geometric data exchange using the IFC standard. Electronic Journal of Information Technology in Construction. ITcon, v. 13, p. 362-380 Special issue Case studies of BIM use, 2008. Disponível em: http://www.itcon.org/2008/24.

RASMUNSSEN, M. H; HVIID, C.A.; KARLSHOJ, J. Web-based topology queries on a BIM model. . In: LINKED DATA IN ARCHITECTURE AND CONSTRUCTION WORKSHOP, 5., 2017, Dijon. Proceedings [... ]. Dijon: LDAC, 2017.

SANTOS, R; COSTA, A. A; GRILO, A. Bibliometric analysis and review of Building Information Modelling literature published between 2005 and 2015. Automation in Construction. v. 80, p. 118-136, 2017. DOI: https://doi.org/10.1016/j.autcon.2017.03.005

SAVIOJA, L; SVENSSON, U. P. Overview of geometrical room acoustic modeling techniques. The Journal of the Acoustical Society of America, v. 138, n. 2, p. 708-730. 2015. DOI:https://doi.org/10.1121/1.4926438

SILVA, J. L.; RIBEIRO, L. A.; MUSSI, A. Q.; SILVA, T. L. Programação em plataforma BIM e a Norma de Desempenho Brasileira: Desenvolvimento de uma aplicação para estimativa de performance acústica em projetos arquitetônicos. In: CONGRESO DE LA SOCIEDAD IBEROAMERICANA DE GRÁFICA DIGITAL, 21, 2017, Concepción. Proceedings [...]. Chile: SIGRADI, 2017. DOI: https://doi.org/10.5151/sigradi2017-061. 
SUNYOUNG, K; COFFEEN, R. C; SANGUINETTI, P. Interoperability Building Information Modeling and acoustical analysis software - A demonstration of a performing arts hall design process. Proceedings of Meetings on Acoustics, v. 19, n. 1, 015136, 2013. DOI:https://doi.org/10.1121/1.4800300

TAN, Y; FANG, Y; ZHOU, T; WANG, Q; CHENG, J. C. P. Improve Indoor Acoustics Performance by Using Building Information Modeling. In: INTERNATIONAL SYMPOSIUM ON AUTOMATION AND ROBOTICS IN CONSTRUCTION, 34., 2017,Taipei. Proceedings [... ]. Oulu: ISARC, 2017. DOI: https://doi.org/10.22260/ISARC2017/0133.

VENUGOPAL, M; EASTMAN, C. M; SACKS R; TEIZER J. Semantics of model views for information exchanges using the industry foundation class schema. Advanced Engineering Informatics, v. 26, n. 2, p. 411- 428, 2012.

DOI:https://doi.org/10.1016/j.aei.2012.01.005

W3C - WORLD WIDE WEB CONSORTIUM. Semantic Web. Disponível em: https://www.w3.org/Consortium/ Acesso em 1 abr. 2019.

WU, C; CLAYTON, M. BIM-Based Acoustic Simulation Framework. In: CIB W78 International Conference, 30., 2013, Beijing. Proceedings [... ]. Beijing: CIB, 2013. Disponível em: http://itc.scix.net/cgi-bin/works/Show?w78-2013-paper66

YALCINKAYA, M, SINGH, V. Patterns and trends in Building Information Modeling (BIM) research: A Latent Semantic Analysis. Automation in Construction, v. 59, p. 68-80, 2015. DOI: https://doi.org/10.1016/j.autcon.2015.07.012

\section{José Luis Menegotto}

Arquiteto. Doutor. Professor da Escola Politécnica da UFRJ. Endereço postal: Av. Athos da Silveira Ramos 149 Bloco D sala D101, Rio de Janeiro, RJ, Brasil, 21941-909.

2 Julio Cesar Boscher Torres

Engenheiro. Doutor. Professor da Escola Politécnica da UFRJ. Endereço postal: Av. Athos da Silveira Ramos 149 Bloco D sala D101, Rio de Janeiro, RJ, Brasil, 21941-909. 